\title{
Requirement of GSK-3 for PUMA induction upon loss of pro-survival PI3K signaling
}

Florian Schubert ${ }^{1,2,3}$, Juliane Rapp ${ }^{1,3}$, Prisca Brauns-Schubert ${ }^{1,2,3,4}$, Lisa Schlicher ${ }^{1,2,3,4}$, Kerstin Stock ${ }^{1,3}$, Manuela Wissler', Martina Weiß ${ }^{1}$, Céline Charvet ${ }^{5}$, Christoph Borner ${ }^{1,2,4}$ and Ulrich Maurer $\mathbb{1}^{1,2,4}$

\begin{abstract}
Growth factor withdrawal induces rapid apoptosis via mitochondrial outer membrane permeabilization. We had previously observed that cell death of IL-3-dependent $\mathrm{Ba} / \mathrm{F3}$ cells, induced by removal of the growth factor, required the activity of the kinase GSK-3. Employing CRISPR/Cas9-mediated gene knockout, we aimed to identify pro-apoptotic GSK-3 regulated factors in this process. Knockout of either Puma or Bim demonstrated that the induction of Puma, but not Bim, was crucial for apoptosis induced by IL-3 deprivation. Thus, we aimed at identifying the GSK-3-dependent PUMA regulator. LOSS of FOXO3A reduced the induction of Puma, while additional loss of p53 completely repressed induction upon growth factor withdrawal. A constitutively active mutant of FOXO3A, which cannot be controlled by AKT directly, still required active GSK-3 for the full transcriptional induction of Puma and cell death upon IL-3 withdrawal. Thus, the suppression of GSK-3 is the key function of PI3K signaling in order to prevent the induction of Puma by FOXO3A and p53 and thereby apoptosis upon growth factor withdrawal.
\end{abstract}

\section{Introduction}

Growth factor signaling supports cell survival through various pathways. Thus, deprivation of growth factor ultimately results in apoptosis. The decisive step for the induction of intrinsic apoptosis is the mitochondrial outer membrane permeabilization (MOMP). This results in the release of cytochrome $\mathrm{c}$ and other proteins from the mitochondrial intermembrane space into the cytosol, leading to apoptosome formation, caspase activation, and apoptosis. MOMP is controlled by proteins of the BCL-2 family. While the pro-apoptotic BCL-2 proteins BAX and BAK are required for the formation of a mitochondrial outer membrane pore, their activity is induced by BH3only proteins (PUMA, BIM, Bid, and others). MOMP is prevented by related proteins with anti-apoptotic function (like BCL-2, MCL-1, BCL-xL) ${ }^{1}$.

\footnotetext{
Correspondence: Ulrich Maurer (ulrich.maurer@mol-med.uni-freiburg.de) ${ }^{1}$ Institute of Molecular Medicine and Cell Research, Faculty of Medicine, AlbertLudwigs-University of Freiburg, Freiburg, Germany

${ }^{2}$ Spemann Graduate School of Biology and Medicine (SGBM), Albert-LudwigsUniversity of Freiburg, Freiburg, Germany

Full list of author information is available at the end of the article

Edited by $G$. Raschellà.
}

MOMP is controlled by growth factor availability, which induces various pathways promoting cell survival. A key pro-survival pathway is the PI3K/AKT signaling pathway, which can prevent MOMP and apoptosis through regulating a number of substrates. For instance, AKT was shown to phosphorylate and inactivate the transcription factor FOXO3A as well as glycogen synthase kinase-3 (GSK-3). The inactivation of both FOXO3A and GSK-3 was shown to play an important role for the pro-survival activity of PI3K/AKT signaling ${ }^{2-4}$. More specifically, it was shown that the suppression of FOXO3A plays an essential role for the suppression of Puma induction and cell death by PI3K signaling ${ }^{5}$.

The death promoting role of GSK-3 is instrumental for p53-mediated Puma induction and apoptosis: GSK-3 phosphorylates the histone acetyl transferase Tip60 (also known as KAT5), which stimulates Tip60 to acetylate p53 at $\mathrm{K} 120$, resulting in the transcriptional induction of Puma and apoptosis upon induction of $\mathrm{p}^{6} 3^{6}$. Interestingly, GSK-3 was also shown to modulate the transcriptional activity of FOXO3A ${ }^{7,8}$.

\section{(c) The Author(s) 2018}

(c) (i) Open Access This article is licensed under a Creative Commons Attribution 4.0 International License, which permits use, sharing, adaptation, distribution and reproduction cc) in any medium or format, as long as you give appropriate credit to the original author(s) and the source, provide a link to the Creative Commons license, and indicate if changes were made. The images or other third party material in this article are included in the article's Creative Commons license, unless indicated otherwise in a credit line to the material. If material is not included in the article's Creative Commons license and your intended use is not permitted by statutory regulation or exceeds the permitted use, you will need to obtain permission directly from the copyright holder. To view a copy of this license, visit http://creativecommons.org/licenses/by/4.0/. 
In the present study, employing knockout by CRISPR/ Cas9, we systematically investigated the role of GSK-3dependent factors required for apoptosis induction by IL3 deprivation. We show that PUMA is the main proapoptotic protein responsible for apoptosis in this context, and that the induction of Puma is mediated by a FOXO3A-, p53-, and GSK-3-dependent mechanism.

\section{Results}

Apoptosis induced by growth factor withdrawal requires GSK-3-dependent PUMA induction

When IL-3-dependent cells such as Ba/F3 or FL5.12 cells (two murine pro B cell lines) are deprived of the growth factor, they undergo rapid apoptosis. Additional treatment with the highly selective GSK-3 inhibitor CT98014 completely blocked IL-3-withdrawal-induced apoptosis of $\mathrm{Ba} / \mathrm{F} 3$ cells as observed previously ${ }^{9}$ (Fig. 1a). We aimed at systematically defining the pro-apoptotic factors involved in IL-3 withdrawal-induced apoptosis and at investigating their link to GSK-3. To address the role of pro-apoptotic BH3-only proteins for growth factorwithdrawal-induced apoptosis, we transduced $\mathrm{Ba} / \mathrm{F} 3$ cells with the lentiCRISPRv2 system targeting either Puma or Bim. As shown in Fig. 1b, apoptosis by IL-3 deprivation was substantially reduced in $\mathrm{Ba} / \mathrm{F} 3$ cells expressing CRISPR/Cas9 targeting Puma, while loss of Bim conferred only moderate protection from cell death. This effect was even more pronounced in the IL-3dependent cell line FL5.12 (Fig. S1A). To further verify the role of PUMA in this system, clones derived from individual cells (single-cell clones) were generated from the CRISPR/Cas9-transduced cultures and cells with frameshift mutations on both Puma alleles or both Bim alleles were selected. Almost all Puma ${ }^{-/-}$single-cell clones were strongly protected from IL-3 withdrawal-induced apoptosis (Fig. 1c) while $\mathrm{Bim}^{-1-}$ single-cell clones exhibited no statistically significant survival advantage (Fig. 1d). The protective effect of Puma depletion lasted at least $24 \mathrm{~h}$, however, the cells committed to apoptosis at later time points. Puma ${ }^{-1-} \mathrm{Ba} / \mathrm{F} 3$ cells additionally treated with the GSK-3 inhibitor CT98014 showed a similar kinetic but the protective effect was more pronounced even after $40 \mathrm{~h}$ (Fig. S1B). Thus, the kinase activity of GSK-3 as well as the induction of Puma are rate-limiting for apoptosis induced by IL-3 withdrawal. The stronger anti-apoptotic effect achieved by inhibition of GSK-3 than by the loss of PUMA alone is consistent with other cell death regulatory factors being regulated by GSK-3, such as MCL- $1^{9}$ (Fig. S1C). We next asked whether PUMA is transcriptionally regulated in a GSK-3-dependent manner upon growth factor withdrawal. Wild-type Ba/F3 cells were deprived of IL-3, treated with GSK-3 inhibitor (CT98014) and Puma mRNA levels were analyzed by quantitative RT-PCR. IL-3 withdrawal-induced Puma
mRNA up to 2-fold after $7.5 \mathrm{~h}$ while Puma mRNA was reduced upon treatment with CT98014 in the absence of IL-3 (Fig. 1e). This effect was reflected by the protein levels of PUMA in Ba/F3 wt cells: PUMA was induced upon IL-3 withdrawal, but this upregulation was completely blocked by addition of CT98014 (Fig. 1f). Loss of PI3K is permitting GSK-3 activity by relieving the suppression of GSK-3 by AKT-mediated phosphorylation. Consistently, we found that the pharmacological inhibition of PI3K resulted in strong induction of PUMA (Fig. S1D).

We next generated IL-2-dependent murine primary lymphocytes, which were deprived of the growth factor. Consistent with the results obtained with IL-3-dependent cell lines, removal of IL-2 induced PUMA and apoptosis, while this was abrogated in presence of the GSK-3 inhibitor (Fig. S1E, Fig. S1F).

We therefore conclude that PUMA represents the main BH3-only protein mediating IL-3 and IL-2 withdrawalinduced apoptosis induction and that the transcriptional induction of Puma is dependent on GSK-3 activity.

\section{p53 has a minor role for GSK-3-dependent PUMA induction}

We next investigated the identity of the transcription factor, which mediates the GSK-3-dependent transcriptional induction of Puma upon loss of growth-factorinduced PI3K signaling. Puma is a crucial pro-apoptotic target of $\mathrm{p} 53^{10,11}$. While $\mathrm{p} 53$ is not stabilized upon growth factor withdrawal, p53 is nevertheless able to induce some of its target genes (such as $M d m 2$ ) when present at very low levels. Thus, even in the absence of DNA damage, we considered p53 as a candidate for GSK-3-dependent Puma induction upon loss of pro-survival signaling during IL-3 withdrawal. To address the role of p53 in this context, we inhibited PI3K by GDC-0941 in HCT116 $p 53^{+/+}$and $p 53^{-1-}$ cells in order to induce PUMA, as described before (see Fig. S1D). As shown in Fig. 2a, inhibition of PI3K-induced PUMA in $p 53^{+/+}$as well as $p 53^{-1-}$ cells, indicating that $\mathrm{p} 53$ is not a major transcription factor responsible for Puma induction in this setting. Consistent with the data shown before, the induction of PUMA was prevented by pharmacological inhibition of GSK-3. To further investigate the role of $\mathrm{p} 53$, we knocked out $p 53$ by CRISPR/Cas9 in Ba/F3 cells, which have an intact p53 signaling pathway ${ }^{6}$. Upon IL-3 withdrawal, $\mathrm{Ba} / \mathrm{F} 3$ cells lacking $p 53$ exhibited some protection from apoptosis (Fig. 2b), which was however not as strong as observed with cells lacking PUMA (see Fig. 1b). We generated $p 53^{-1-}$ single-cell clones and subjected them to IL-3 deprivation. We unexpectedly observed that individual $\mathrm{Ba} / \mathrm{F} 3$ cell clones lacking $p 53$ exhibited quite some variation regarding the protection from IL-3 withdrawal-induced apoptosis. Nevertheless, on average, the protection conferred by lack of p53 was 

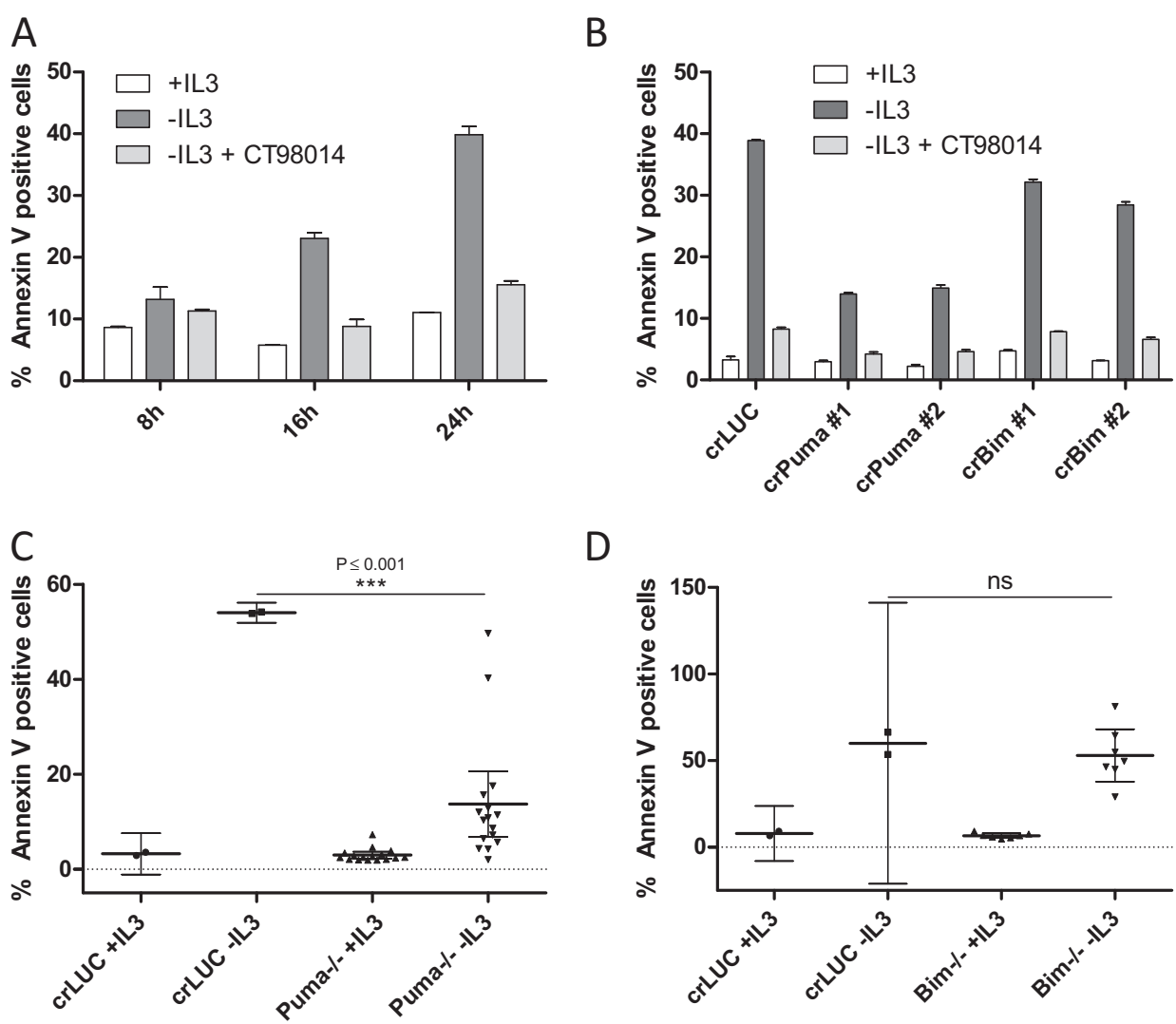

D
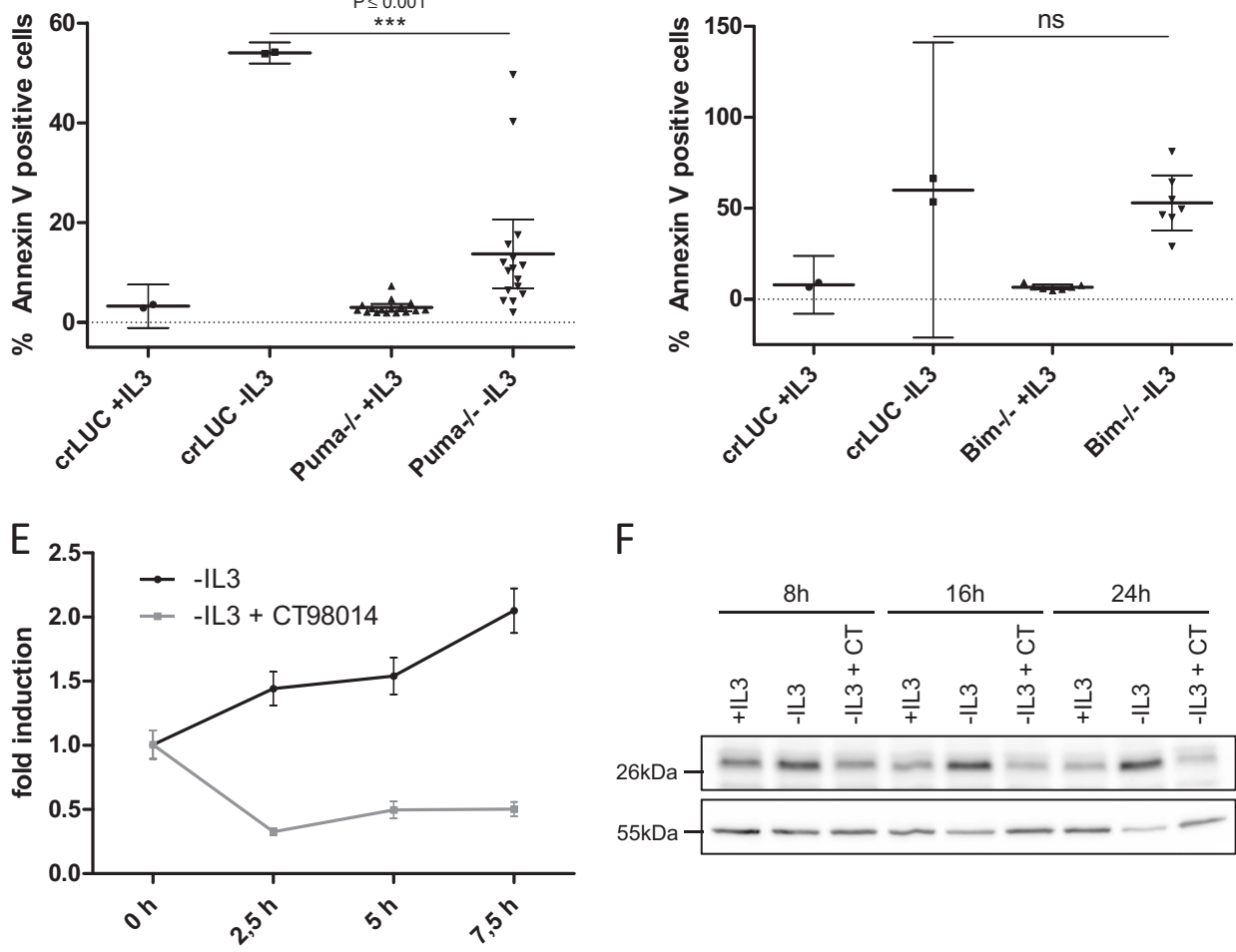

$\mathrm{F}$

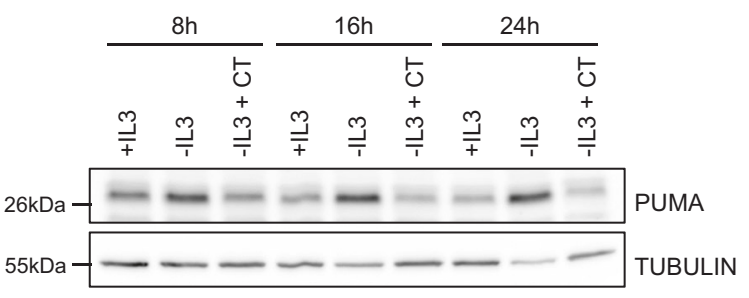

Fig. 1 Apoptosis induced by growth factor withdrawal requires GSK-3-dependent PUMA induction. a Ba/F3 cells were deprived of IL-3 in the presence or absence of CT98014 (0.75 $\mu \mathrm{M})$ and analyzed for apoptosis by Annexin V staining and flow cytometry analysis. Error bars represent SD from technical replicates. b Ba/F3 cells expressing CRISPR/Cas9 targeting Luciferase (crLUC), Puma (crPuma), or Bim (crBim) were deprived of IL-3 in presence or absence of $\mathrm{CT} 98014(0.75 \mu \mathrm{M})$ and analyzed for apoptosis by Annexin $\mathrm{V}$ staining after $18 \mathrm{~h}$. Error bars represent SD from technical replicates. c Ba/F3 Puma ${ }^{-1-}$ single-cell clones and Ba/F3 expressing CRISPR/Cas9 constructs targeting Luciferase were deprived of IL-3 for $18 \mathrm{~h}$ and analyzed for apoptosis by Annexin $\vee$ staining. Each dot represents the mean of two independent experiments analyzing an individual single-cell clone. Error bars represent 95\% confidence interval from two independent experiments $(n=2)$. Significance was tested by one-way ANOVA with post hoc Tukey's multiple comparison test. $\mathbf{d}$ Ba/F3 $\mathrm{Bim}^{-/-}$single-cell clones and Ba/F3 expressing CRISPR/Cas9 constructs targeting Luciferase were deprived of IL-3 for $18 \mathrm{~h}$ and analyzed for apoptosis by Annexin $\mathrm{V}$ staining. Each dot represents the mean of two independent experiments analyzing an individual single-cell clone. Error bars represent the 95\% confidence interval from two independent experiments $(n=2)$. Significance was tested by one-way ANOVA with post hoc Tukey's multiple comparison test. ns = not significant. e Ba/F3 cells were deprived of IL-3 in presence or absence of CT98014 $(0.75 \mu \mathrm{M})$ and harvested after the indicated time. The $0 \mathrm{~h}$ condition represents cells kept in medium with IL-3. RNA levels were analyzed by qRT-PCR with primers for Puma and $L 32$ as internal reference. Data points show relative (mRNA) Puma induction compared to cells kept in medium with IL-3. Error bars represent SD from technical replicates. $\mathbf{f}$ Cells from a were subjected to western blotting and analyzed with the antibodies indicated. 

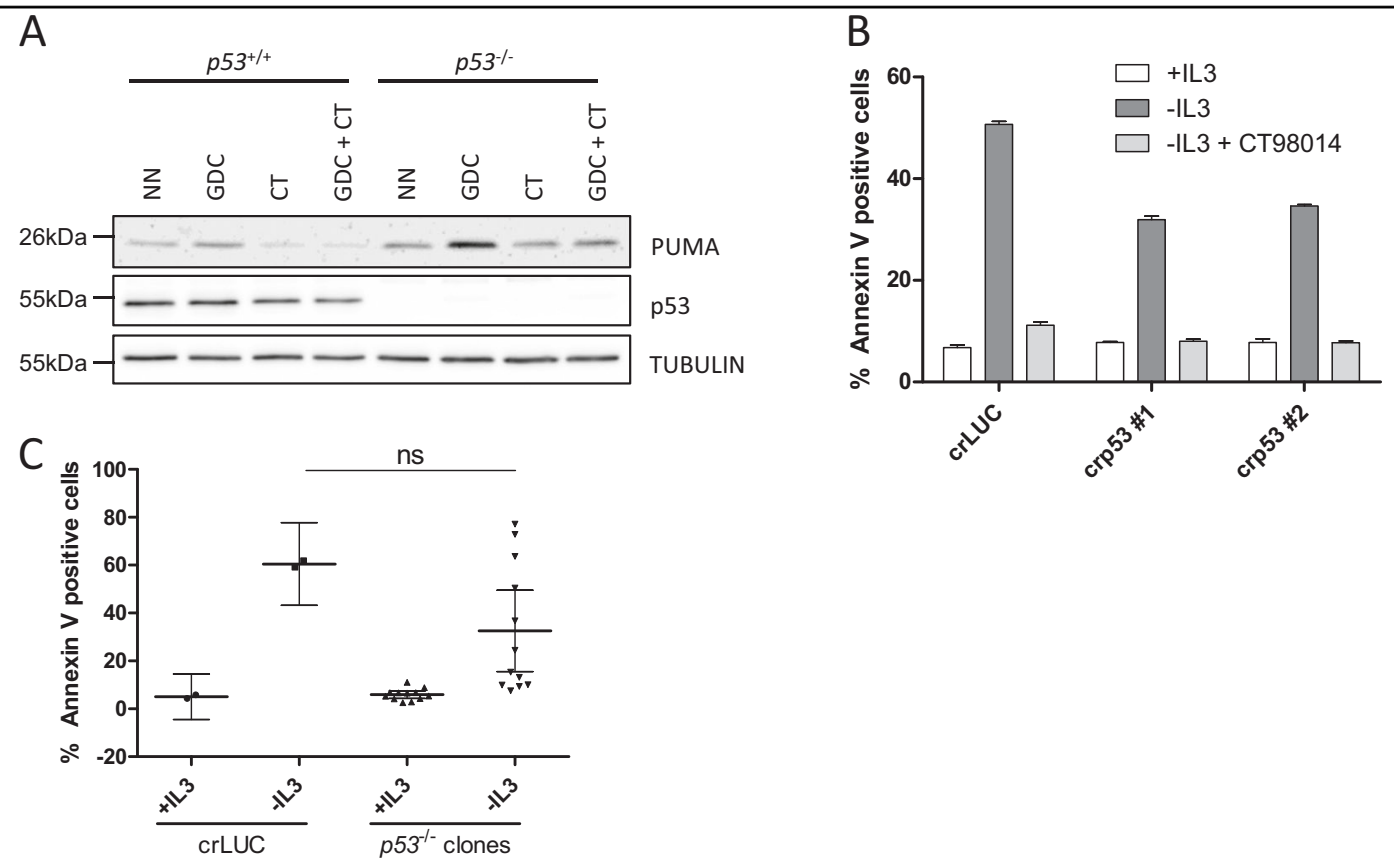

D
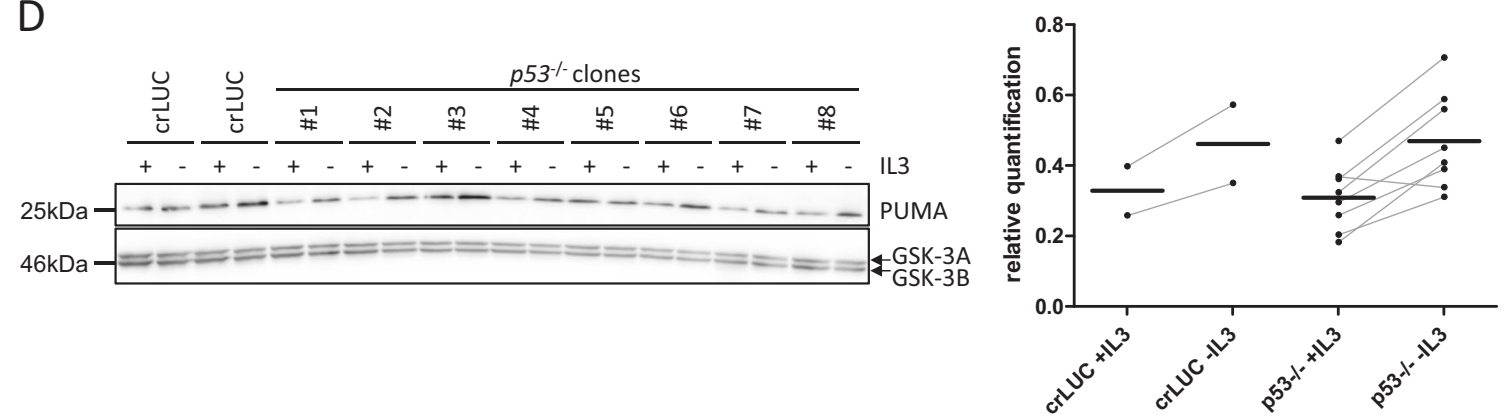

$\mathrm{E}$

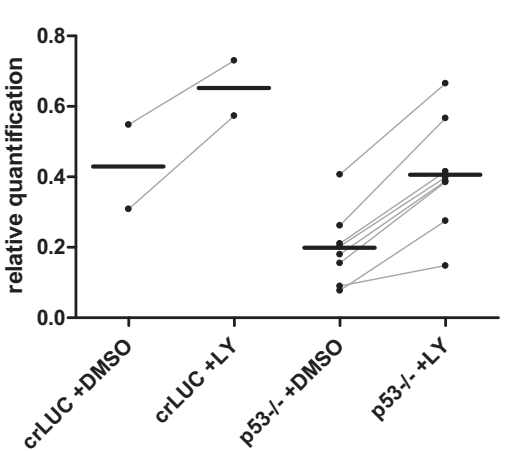

Fig. 2 p53 has a minor role for GSK-3-dependent PUMA induction. a HCT116 $p 53^{-/-}$or $p 53^{+/+}$were treated with GDC-0941 (GDC, $10 \mu M$ ), CT98014 $(C T, 0.75 \mu \mathrm{M})$, or a combination of both for $7 \mathrm{~h}$. The cells were harvested, subjected to western blotting and analyzed by the antibodies indicated. b Ba/F3 expressing CRISPR/Cas9 targeting $p 53$ or Luciferase were deprived of IL-3, in presence or absence of CT98014 (0.75 $\mu \mathrm{M})$ for $18 \mathrm{~h}$ and analyzed for apoptosis by Annexin $\mathrm{V}$ staining. Error bars represent SD from technical replicates. c Ba/F3 $p 53^{-/-}$single-cell clones and Ba/F3 expressing CRISPR/Cas 9 constructs targeting Luciferase were deprived of $\mathrm{IL}-3$ for $18 \mathrm{~h}$ and analyzed for apoptosis by Annexin $\mathrm{V}$ staining. Each dot represents the mean of two independent experiments analyzing an individual single-cell clone. Error bars represent 95\% confidence interval from two independent experiments $(n=2)$. Significance was tested by one-way ANOVA with post hoc Tukey's multiple comparison test. $\mathrm{ns}=$ not significant. $\mathbf{d}$ Ba/F3 p53 ${ }^{-1-}$ single-cell clones and two independent cell lines of Ba/F3 cells infected with CRISPR/Cas9 targeting Luciferase were deprived of IL-3 for $12 \mathrm{~h}$, harvested and analyzed by western blotting by probing with antibodies as indicated. Bands were quantified using FusionCapt Advance Solo 4 16.08. PUMA levels were normalized to GSK-3 levels (loading control). e Ba/F3 $p 53^{-1-}$ single-cell clones and two independent cell lines of Ba/F3 expressing CRISPR/Cas9 constructs targeting Luciferase were treated with LY294002 $(10 \mu \mathrm{M})$ or DMSO (-) for $18 \mathrm{~h}$, harvested and analyzed by western blotting with the antibodies indicated. Bands were quantified using FusionCapt Advance Solo 4 16.08. PUMA levels were normalized to GSK-3 levels (loading control). 
modest (Fig. 2c). Interestingly, a variation in IL-3-induced apoptosis was also observed in different single-cell clones expressing CRISPR/Cas9 targeting Luciferase and-of note-in wild-type $\mathrm{Ba} / \mathrm{F} 3$ cells, indicating that individual clones always seems to exhibit some variation, even in the absence of previous manipulation. We therefore always analyzed a high number of clones (Fig. S2A+B). Consistent with the modest protection of $p 53^{-1-}$ single-cell clones from IL-3 deprivation, PUMA induction was still observed in $p 53^{-/-}$single-cell clones upon IL-3 withdrawal (Fig. 2d) or PI3K inhibition by LY294002 (Fig. 2e) and was still dependent on GSK-3 (Fig. S2C). However, the quantification of PUMA expression levels revealed that, while Puma was still induced, the PUMA protein levels were generally somewhat reduced in $p 53^{-1-}$ cells (Fig. 2e, right). Together, although there is a GSK-3dependent effect of p53 on PUMA and apoptosis induction, p53 seems not to be the main GSK-3-dependent transcription factor responsible for the induction of PUMA and apoptosis upon IL-3 withdrawal.

\section{FOXO3A is an important Puma inducer upon growth factor deprivation}

To test other transcription factors for their role in inducing Puma upon IL-3 deprivation, we generated $\mathrm{Ba} /$ F3 expressing CRISPR/Cas9 targeting Foxo1, Foxo3a, and the p53 relatives $p 63$ and $p 73$. We then subjected these cells to IL-3 withdrawal and analyzed apoptosis induction. Among the cells we tested, only Foxo3a-targeted cells showed a protection from IL-3 deprivation (Fig. 3a, Fig. S3A). Single-cell clones generated from these cells again showed a wide distribution of sensitivity to IL-3 withdrawal but were on average significantly protected compared to control cells (Fig. 3b). The Foxo3a ${ }^{-1-}$ singlecell clones showed a diminished capacity to induce PUMA upon IL-3 withdrawal (Fig. S3B). Because of the high variation of individual Foxo3a $a^{-1-}$ single-cell clones to induce PUMA, they were pooled and we observed a clear reduction of the signal for PUMA upon IL-3 withdrawal (Fig. 3d). Likewise, Foxo3a $a^{-1-}$ single-cell clones treated with the PI3K inhibitor LY294002 exhibited reduced Puma induction, as evident from the quantification of the PUMA expression levels (Fig. 3d).

\section{Double knockout of Foxo3a and $p 53$ fully protects cells} from IL-3-induced apoptosis and prevents Puma induction

We next addressed the combined role of p53 and FOXO3A to induce apoptosis and PUMA upon IL-3 withdrawal. We generated cells expressing CRISPR/Cas9 targeting p53 and Foxo3a simultaneously and identified two single-cell clones with frameshift mutations on both alleles of each $p 53$ and Foxo3a (double knock out, DKO). As shown in Fig. 4a, both DKO clones were protected from IL-3 deprivation-induced apoptosis. In line with this,
PUMA induction was absent upon treatment with LY294002 (Fig. 4b) or IL-3 withdrawal (Fig. 4c) in these cells. Thus, p53 and FOXO3A together are required for PUMA upregulation and apoptosis upon IL-3 withdrawal, possibly also compensating for each other to some extent in cells lacking either p53 or FOXO3A.

\section{The induction of Puma by FOXO3A depends on GSK-3}

To confirm the major role of FOXO3A and its GSK-3 dependency in IL-3 withdrawal-induced apoptosis, we reexpressed FOXO3A with silent mutations, rendering it resistant to CRISPR/Cas9 cleavage, in a Ba/F3 bulk culture which expressed a CRISPR/Cas9 construct targeting Foxo3a (Fig. 5a) as well as in a Foxo3 $a^{-1-}$ single-cell clone (Fig. 5b). Re-expression of FOXO3A re-established apoptosis induction to a comparable level as in control cells transduced with FOXO3A in bulk culture and the single-cell clone (Fig. 5a, b). Importantly, restoring FOXO3A and thereby the competence to undergo apoptosis did not relieve the requirement for GSK-3 activity, as inhibition of GSK-3 suppressed apoptosis induced by IL-3 withdrawal in FOXO3A overexpressing cells. Thus, FOXO3A-dependent PUMA and apoptosis induction upon growth factor withdrawal requires GSK-3. Of note, the induction of apoptosis as well as the induction of PUMA in Foxo3a $a^{-l-}$ cells was still dependent on GSK-3 (Fig. S4A+B), confirming that the regulation of PUMA by p53 is also controlled by GSK- $3^{6}$.

While PUMA protein levels were reduced in empty vector-transduced Foxo3 $a^{-1-}$ cells upon IL-3 withdrawal, PUMA induction was restored in Foxo $3 a^{-1-}$ cells reexpressing CRISPR/Cas9-resistant FOXO3A (Fig. 5c). Consistently, FOXO3A overexpression in HCT116 $p 53^{-1-}$ cells increased PUMA induction in cells treated with the AKT inhibitor GDC-0941 (Fig. 5d). We therefore conclude that FOXO3A induces Puma when Ba/F3 are deprived of IL-3, while p53 contributes to this induction, and that both transcription factors require GSK-3 activity for the induction of PUMA and apoptosis.

\section{FOXO3A requires GSK-3 activity for full transcriptional activity}

As shown above, the pharmacological inhibition of GSK-3 was sufficient to prevent growth factor withdrawal-induced PUMA induction and apoptosis. We previously demonstrated that when p53 is stabilized by DNA damage, GSK-3 activity regulates the capacity of p53 to induce Puma by phosphorylating Tip60 at S86, which stimulates Tip60 to acetylate lysine 120 (K120) of p53 ${ }^{6}$. K120-acetylated p53 then induces Puma which leads to apoptosis $^{12-14}$. However, in $p 53^{-/-}$cells we observed FOXO3A-dependent PUMA induction and apoptosis, which was also dependent on GSK-3 activity (Figs. 2a-c and $5 \mathrm{~d}$ ). To investigate the role of GSK-3 for the 

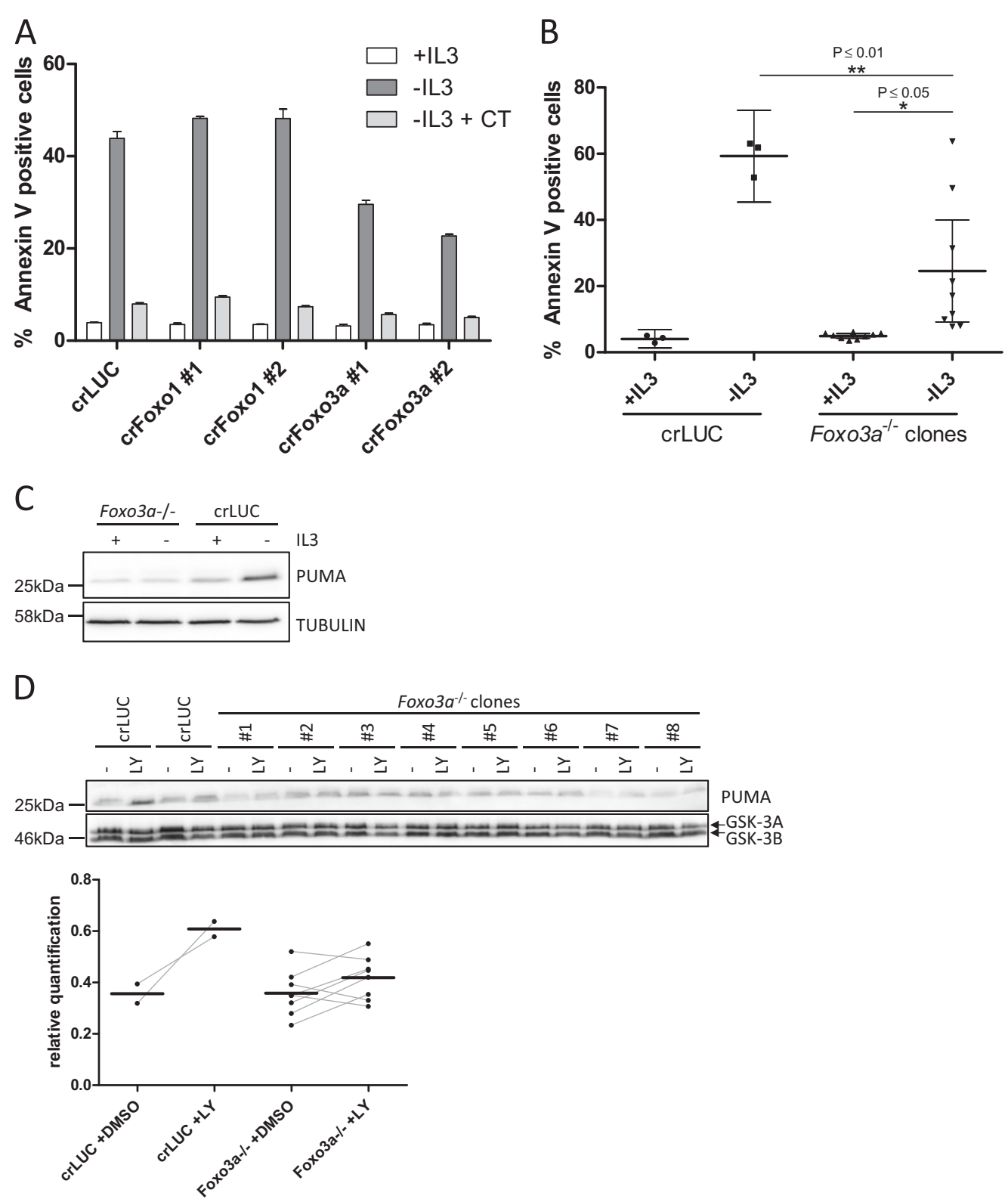

Fig. 3 FOXO3A is an important Puma inducer upon growth factor deprivation. a Ba/F3 expressing CRISPR/Cas9 targeting Foxo3a, Foxo1, or Luciferase were deprived of IL-3 in the presence or absence of CT98014 (0.75 $\mu \mathrm{M})$ for $18 \mathrm{~h}$ and analyzed for apoptosis by Annexin $\mathrm{V}$ staining. $\mathbf{b}$ Ba/F3 FoxO3 $a^{-1-}$ single-cell clones and Ba/F3 expressing CRISPR/Cas9 constructs targeting Luciferase were deprived of IL-3 for $18 \mathrm{~h}$ and analyzed for apoptosis by Annexin $V$ staining. Each dot represents the mean of three independent experiments analyzing an individual single-cell clone. Error bars represent 95\% confidence interval from three independent experiments $(n=3)$. Significance was tested by one-way ANOVA with post hoc Tukey's multiple comparison test. $\mathbf{c}$ Equal cell numbers of eight different Ba/F3 Foxo3a ${ }^{-1-}$ single-cell clones were pooled. This pool and Ba/F3 expressing CRISPR/Cas9 targeting Luciferase were deprived of IL-3 for $9 \mathrm{~h}$, harvested and analyzed by western blotting with the antibodies indicated. $\mathbf{d}$ Ba/F3 Foxo3a $a^{-1-}$ single-cell clones and two independent cell lines of Ba/F3 expressing CRISPR/Cas9 constructs targeting Luciferase were treated with LY294002 (10 $\mu \mathrm{M})$ or DMSO (-) for $18 \mathrm{~h}$, harvested and analyzed by western blotting with the antibodies indicated. Bands were quantified using FusionCapt Advance Solo 4 16.08. PUMA levels were normalized to GSK-3 levels (loading control).

transcriptional regulation of Puma by FOXO3A, we generated a promoter reporter system where Luciferase expression is under control of the Puma promoter, which includes a binding site for FOXO3A ${ }^{5}$. It is well established that FOXO3A is controlled by the PI3K/AKT pathway as phosphorylation of FOXO3A residues T32, S253, and S315 by AKT results in retention of the transcription factor in the cytosol ${ }^{2}$. We expressed a FOXO3A-triple mutant (TM; T32A-S253A-S315A), which cannot be phosphorylated by $\mathrm{AKT}$ and is considered to be 

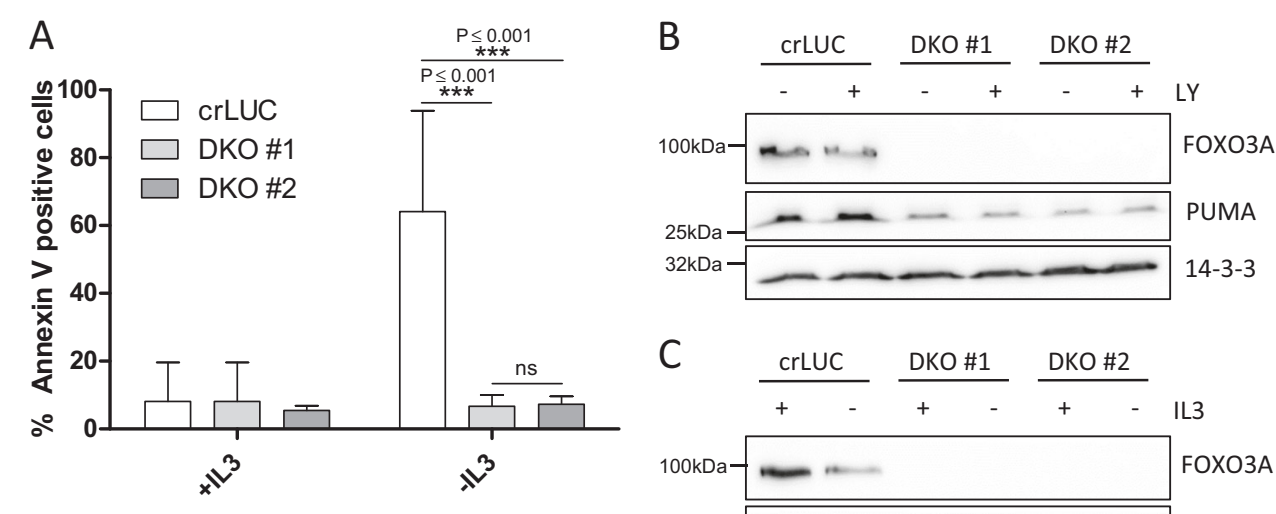

C

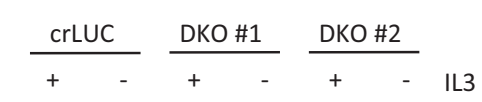

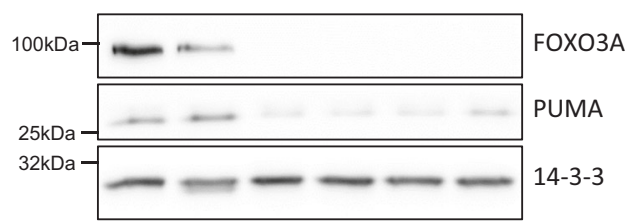

Fig. 4 Double knockout of Foxo3a and $p 53$ fully protects cells from IL-3-induced apoptosis and prevents Puma induction. a Ba/F3 $p 53^{-/-}$ FoxO3 $a^{-1-}$ single-cell clones (DKO \#1 and DKO \#2) and Ba/F3 expressing CRISPR/Cas9 constructs targeting Luciferase (crLUC) were deprived of IL-3 for $18 \mathrm{~h}$ and analyzed for apoptosis by Annexin $\mathrm{V}$ staining. Error bars represent 95\% confidence intervals from four independent experiments $(n=4)$. Significance was tested by one-way ANOVA with post hoc Tukey's multiple comparison test. ns = not significant. ${ }^{* *} P<0,001$. $\mathbf{b}$ The same cells were treated with LY294002 $(10 \mu \mathrm{M})$ for $18 \mathrm{~h}$ or left untreated. The cells were harvested after the treatment and subjected to western blotting. The protein levels were analyzed by the antibodies indicated. c The same cells were deprived of IL-3 for $18 \mathrm{~h}$, harvested after the treatment and subjected to western blotting.

constitutive active, along with the Puma promoter reporter constructs. As shown in Fig. 6a, overexpression of FOXO3A-TM induced Luciferase expression controlled by the Puma promoter. This was dependent on a FOXO3A binding site in the promoter region, as mutation of the binding site for FOXO3A prevented Luciferase induction. However, when we added the GSK-3 inhibitor CT98014, the ability of FOXO3A-TM to induce Luciferase through the Puma promoter was substantially decreased. We therefore conclude that FOXO3A, in order to fully exhibit its transcriptional activity at the Puma promoter, requires not only the dephosphorylation of AKT phosphorylation sites in FOXO3A, but also the activity of GSK-3.

We next established $\mathrm{Ba} / \mathrm{F} 3$ cell lines overexpressing FOXO3A-TM. We expressed FOXO3A-TM with silent mutations, mediating resistance to CRISPR/Cas9 cleavage, in a $\mathrm{Ba} / \mathrm{F} 3$ bulk culture expressing a CRISPR/Cas9 construct targeting Foxo3a, or in control cells with CRISPR/Cas9 targeting Luciferase. This led to a slightly increased background of apoptosis even when IL-3 was available. However, although FOXO3A-TM is considered to be constitutively active, IL-3 deprivation was required to induce apoptosis (Fig. 6b, left). Importantly, apoptosis could be inhibited by addition of CT98014 (Fig. 6b, right), showing that FOXO3A-TM (which is not controlled by AKT) requires GSK-3 activity to induce apoptosis upon IL-3 withdrawal. As the inhibition of GSK-3 did not affect the binding to 14-3-3 proteins (Fig. 6c) or the nuclear localization of FOXO3A (Fig. S5A), the regulation of
FOXO3A-mediated transcription by GSK-3 is independent of FOXO3A translocation. Interestingly, we observed an interaction of GSK-3 with FLAG-tagged FOXO3A overexpressed in 293T HEK cells (Fig. 6c). Together, the results show that activity of GSK-3 is decisive for the FOXO3A and p53-mediated PUMA induction in the absence of PI3K signaling.

\section{Discussion}

We had previously shown that GSK-3 phosphorylates the anti-apoptotic protein MCL-1 upon growth factor withdrawal or PI3K inhibition, which regulates lymphocyte survival $^{9,15}$. In this study, we aimed at systematically identifying the GSK-3-dependent pro-apoptotic factors, promoting apoptosis upon depletion from growth factor. We showed that PUMA rather than BIM mediates IL-3 withdrawal-induced apoptosis, although both proteins were upregulated upon IL-3 deprivation (data not shown). In line with our data, it was previously shown that PUMA and BIM are induced by IL-2 withdrawal ${ }^{5,16}$. Our findings also confirm a previous report that IL-3-dependent myeloid cell lines from mice lacking PUMA, but not BIM, were protected from IL-3 withdrawal-induced apoptosis $^{17}$.

FOXO3A and p53 were both described to be important for growth factor withdrawal-induced apoptosis. FOXO3A deficiency can protect cells from cytokine withdrawal-induced apoptosis ${ }^{16,18}$, and FOXO3A was shown to be crucial for PUMA induction upon growth factor withdrawal ${ }^{5}$. It was also found that $\mathrm{p} 53$, FOXO and 


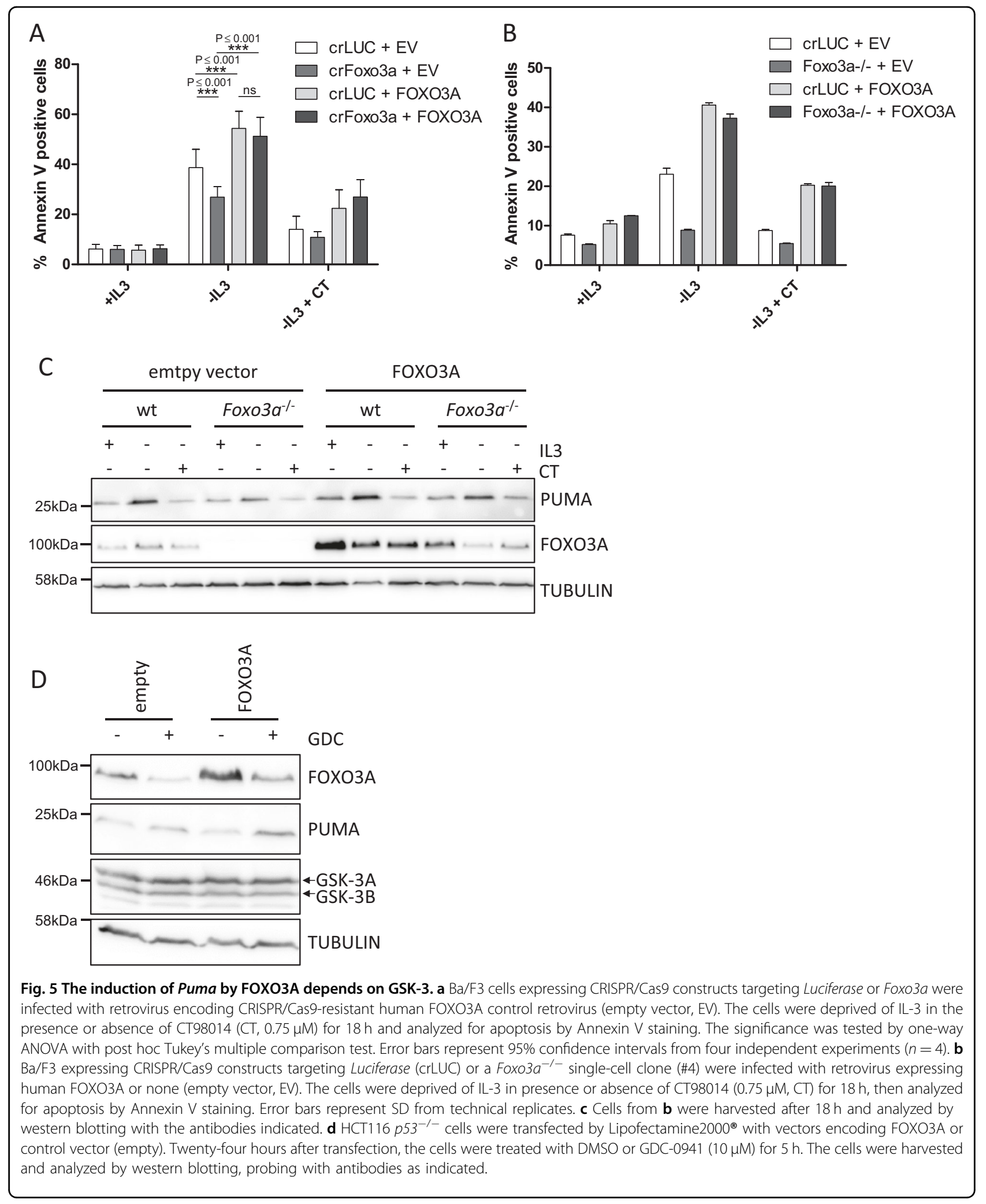



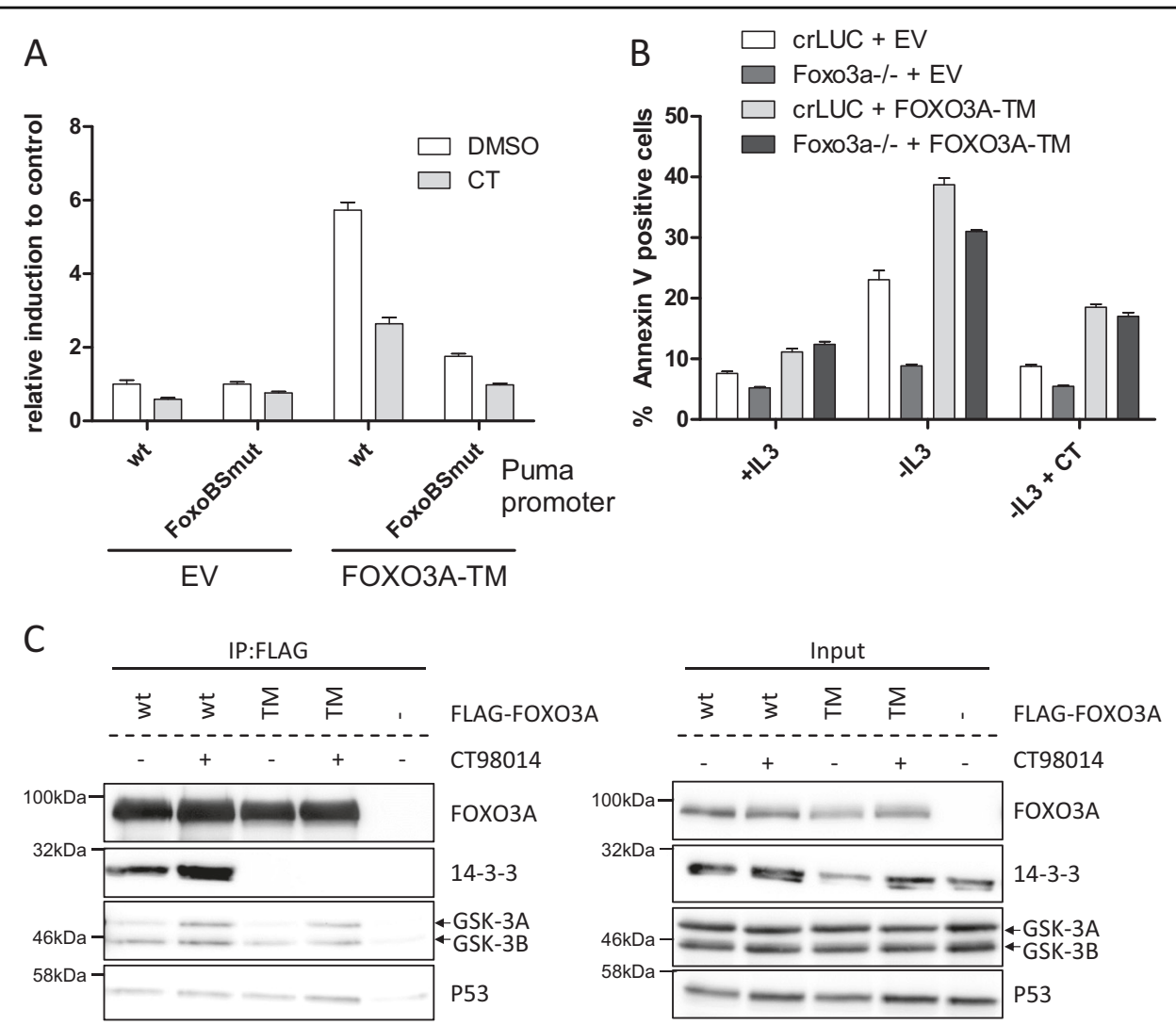

Fig. 6 FOXO3A requires GSK-3 activity for full transcriptional activity. a 293T HEK cells were transfected with a reporter plasmid with Luciferase driven by a wild type (wt) or FOXO3A binding site mutant (FoxoBSmut) Puma promoter fragment, a Renilla reporter plasmid as internal control and a construct encoding FOXO3A-TM or empty vector. Eight hours after transfection, the cells were treated with CT98014 (CT, $0.75 \mu \mathrm{M}$,$) or DMSO for 18 \mathrm{~h}$. Luciferase activity was analyzed and normalized to Renilla activity. Error bars represent SD from technical replicates. b Ba/F3 cells expressing CRISPR/ Cas9 constructs targeting Luciferase or a Foxo3a ${ }^{-1-}$ single-cell clone were infected with retrovirus expressing human FOXO3A-TM or none (empty vector, EV). The cells were deprived of IL-3+/- CT98014 (CT, $0.75 \mu \mathrm{M})$ for $18 \mathrm{~h}$, then stained with Annexin-V-FITC and analyzed by flow cytometry. Error bars represent SD from technical replicates. This experiment was done together with the one shown in Fig. 5b, and the controls are identical. $\mathbf{c}$ HEK 293T cell were transfected with constructs encoding wild-type, FLAG-tagged FOXO3A (wt) or FLAG-tagged FOXO3A-TM (TM) or control vector (-). Eight hours later, the cells were treated with CT98014 $(0.75 \mu \mathrm{M})$ or left untreated for $18 \mathrm{~h}$. The cells were lysed and $2 \%$ of the lysate were kept as input. FLAG immunoprecipitation was performed with the remaining lysate. After washing and elution with 3xFLAG peptide, eluate and input fractions were analyzed by western blotting with the antibodies indicated.

E-box-binding transcription factors share many targeted genes in response to PI3K inhibition in Rat-1 cells and a dominant-negative p53 resulted in partial resistance to apoptosis upon PI3K inhibition ${ }^{19}$. Likewise, HoxB8transformed factor-dependent myeloid (FDM) cells from $\mathrm{p} 53^{-/-}$mice were less sensitive for IL-3 deprivationinduced cell death and showed reduced PUMA induction. In this study, FDM cells from FOXO3A ${ }^{-1-}$ mice showed an even higher susceptibility to cell death after IL-3 $\operatorname{loss}^{20}$. The same authors reported that PUMA induction was independent of PI3K, a finding which is not confirmed by our data ${ }^{17,21}$. Instead, we found that inhibition of the PI3K/AKT pathway upregulated PUMA in $p 53^{-1-}$ HCT116 cells and Ba/F3 cells.

In addition, p53 negative Ba/F3 cells were fully protected from apoptosis only when GSK-3 was inhibited, which further supports the important apoptosisregulatory role of the PI3K/AKT pathway also in absence of $\mathrm{p} 53$.

We have shown that the induction of Puma by FOXO3A and/or p53 is dependent on GSK-3 activity. Importantly, we observed that even the transcriptional activity of a FOXO3A-TM mutant, which is considered constitutively active due to the loss of the AKT phosphorylation sites, required GSK-3. Our results suggest that the inhibition of GSK-3 is the key pro-survival function of PI3K signaling, being more important than the inactivation of FOXO3A. In line with this finding, full transcriptional activity of Foxo1/3/4 was shown to require GSK-3, as the induction of IGF-IR gene by serum starvation or AKT inhibition required both active GSK-3 and Foxo1/3/4 ${ }^{8}$. Supporting a PI3K-dependent regulation of 
FOXO3A independent of the AKT phosphorylation sites, the DNA-binding and transcriptional activity of a triple $\mathrm{S} /$ A mutant of the Foxo homolog in C. elegans, DAF-16, which is considered constitutive active, was shown to be further increased by PI3K inhibition by LY294002 and Wortmannin ${ }^{22}$. Interestingly, promotion of FOXO3A activity to induce PUMA by GSK-3 was independent of the interaction of FOXO3A with 14-3-3 or its subcellular localization.

It is not clear however, how GSK-3 and FOXO3A cooperate. In a study of Terragni et al., upregulated genes upon inhibition of PI3K were co-regulated by FOXO3A, MITF, and USF1, with the latter two being regulated by GSK $-3^{23}$. Another explanation for the functional interaction of FOXO3A and GSK-3 could be provided by the regulation of TIP60. We could previously demonstrate the requirement of GSK-3 for Puma induction by p53 in the context of DNA damage and subsequent p53 stabilization by the phosphorylation of the histone acetyl transferase Tip60 (KAT5). Increased KAT activity of Tip60 is induced by GSK-3 mediated phosphorylation, resulting in the acetylation of p53 in the DNA-binding domain at lysine 120 , which enables p53 to promote the transcription of Puma. It is possible that GSK-3 activated Tip60 acts on chromatin or FOXO3A directly, thereby promoting transcriptional Puma induction ${ }^{6}$.

A direct phosphorylation of FOXO3A by GSK-3 would also be a possibility as to how GSK- 3 directly promotes the transcriptional activity of FOXO3A, which would be consistent with our data demonstrating a cooperation of FOXO3A-TM and GSK-3 for the regulation of the Puma promoter.

Together, in this study, we provide evidence that Puma induction by FOXO3A is an important step in IL-3 withdrawal-induced apoptosis, and that the Puma inducing function of FOXO3A is dependent on GSK-3 activity. Thus, GSK-3 is crucial for the full activation of FOXO3A transcriptional activity when the PI3K pathway is not active. As pharmacological modulation of kinase signaling pathways is a promising strategy for cancer therapy, a more detailed understanding of the underlying mechanisms will improve defining the crucial targets.

\section{Materials and methods}

\section{Reagents and antibodies}

LY294002 was from Sigma-Aldrich (St. Louis, MO, USA), GDC-0941 and CT98014 were from Axon Medchem (Groningen, Netherlands), Annexin-V-FITC was generated in our lab. Annexin-V-APC (RUO) was from Becton Dickinson (Franklin Lakes, NJ, USA). 4-OHT was from Sigma-Aldrich (St. Louis, MO, USA).

IL-2 and IL-3 were from Peprotech (Rocky Hill, NJ, USA).

The following antibodies were used for western blotting: Puma (\#3043) was from Prosci (Poway, CA, USA), human
Table 1 gRNAs

\begin{tabular}{lll}
\hline Target & gRNA name & gRNA sequence (20xN-NGG) \\
\hline p53 Exon3 & p53 gRNA \#1 (759) & AGTGAAGCCCTCCGAGTGTC-AGG \\
p53 Exon3 & p53 gRNA \#2 (760) & AGGAGCTCCTGACACTCGGA-GGG \\
p53 Exon3 & p53 gRNA \#3 (761) & GACACTCGGAGGGCTCACT-TGG \\
p63 Exon3 & p63 gRNA \#1 & TCCACAAAGTTCAACTCGAT-GGG \\
p63 Exon4 & p63 gRNA \#2 & CCGTCACGCTATTCTGTGCG-TGG \\
p63 Exon4 & p63 gRNA \#3 & AGCCCCAGGTTCGTGTACTG-TGG \\
p73 Exon3 & p73 gRNA \#1 & CCGGGGTAGTCGGTATTGGA-AGG \\
p73 Exon3 & p73 gRNA \#2 & CGGGGTGTAGGGGCTCGCCG-GGG \\
Puma Exon1 & Puma gRNA \#1 & ATGGCCCGCGCACGCCAGGA-GGG \\
Puma Exon1 & Puma gRNA \#2 & AGCTCTCCGGAGCCCGTAGA-GGG \\
Puma Exon1 & Puma gRNA \#3 & GGAAGGGGCGCGACTGTCG-CGG \\
Bim Exon1 & Bim gRNA \#1 & ACTTACATCAGAAGGTTGCT-TGG \\
Bim Exon1 & Bim gRNA \#2 & TTGCGGTTCTGTCTGTAGGG-AGG \\
Foxo1 Exon1 & Foxo1 gRNA \#1 & TCGTCGCGCCGCAACGCGTG-GGG \\
Foxo1 Exon1 & Foxo1 gRNA \#2 & GGAGAGTGAGGACTTCGCGC-GGG \\
Foxo3a Exon1 & Foxo3a gRNA \#1 & CACGCCGCCACCGATCACCA-TGG \\
Foxo3a Exon1 & Foxo3a gRNA \#2 & TCTCGATGGCGCGGGTGATC-AGG \\
Luciferase & Luciferase gRNA & ACCGCTCCGGCGAAGGCGA-NGG \\
\hline
\end{tabular}

Puma (\#12450), Foxo3a (\#2497) were from Cell Signaling Technologies (Danvers, MA, USA). GSK3a/b (sc-56913), 14-3-3 (sc-1657) and NFATc1 (sc-7294) were from Santa Cruz (Dallas, TX, USA). Tubulin (MCA77G) was from Bio-Rad (Hercules, CA, USA). MCL1 (600-401-394S) was from Rockland (Limerick, PA, USA).

FLAG-M2 agarose affinity beads were from SigmaAldrich (St. Louis, MO, USA).

\section{Generation of ko cell lines using lentiviral CRISPR/Cas9}

The lentiCRISPRV2 system was used to generate ko cells as described by others ${ }^{24}$. At least two different guide RNAs for each target were designed using crispr.mit.edu and a guide RNA targeting Luciferase was designed as control (see Table 1: gRNAs). The guide RNAs were cloned into the lentiCRISPRV2 plasmid (a gift from Feng Zhang, Addgene plasmid \# 52961) and lentiviral particles were produced by transfection of $5 \mu \mathrm{g}$ lentiCRISPRv2, 1.5 $\mu \mathrm{g}$ pMISSIONVSV-G (Sigma-Aldrich) and $3 \mu \mathrm{g}$ pMISSION GAG POL (Sigma-Aldrich) with Attractene (Quiagen, Hilden, Germany) into 293T HEK cells seeded at $25 \%$ confluency in a $78-\mathrm{cm}^{2}$ culture plate the day before transfection. The morning after transfection, fresh medium was added. In the evening, $4 \mathrm{ml}$ medium was added. The next day, viral supernatants were harvested, filtered $(0,45 \mu \mathrm{M})$ and supplemented with $5 \mu \mathrm{g} / \mathrm{ml}$ polybrene 
Table 2 Primers

\begin{tabular}{ll}
\hline Primer & Sequence (5' -3') $^{\prime}$ \\
\hline p53 for & TGTCTGTAAATCCTGCGGGG \\
p53 rev & GAGGCTAAAAAGGTCAGGGC \\
p63 Exon3 for & TAAGACGGTGAGCCACTCCA \\
p63 Exon3 rev & CCCACTGCAGAAAGCTGAGA \\
p63 Exon4 for & GATGGGTGGCTTAATTGGGA \\
p63 Exon4 rev & ACACACCCTGGAACCTGTCT \\
p73 for & CTACTCACTGTCCAGGTGGC \\
p73 rev & ACAAGTAGTGGCCTGTTGGG \\
Puma for & TTCCTGGGTGGAGTGACTT \\
Puma rev & AGGGACTTCCCACTCGACTT \\
Bim for & ACGAAATGTAGACGTCCCGC \\
Bim rev & CCCACAGCCTTGAACCGAT \\
Foxo1 for & AACCAGTCCAACTCGACCAC \\
Foxo1 rev & AAGTTCCCAAACGAGCCCTG \\
Foxo3a for & GGAGAGAGCAAGAGCCCAAG \\
Foxo3a rev & GACCCTCCCTTCCCACTTG \\
qRT-PCR Puma for & GCCCAGCAGCACTTAGAGTC \\
qRT-PCR Puma rev & GGTGTCGATGCTGCTCTTCT \\
qRT-PCR L32 rev & TTAAGCGAAACTGGCGGAAC \\
\hline
\end{tabular}

(Sigma-Aldrich). The target cells were infected by spinfection at $400 \times g$ for $10 \mathrm{~min}$. The following day, selection was started using $4 \mu \mathrm{g} / \mathrm{ml}$ puromycin (Sigma-Aldrich) for at least 3 days and until no viable cells were detected in an uninfected control. Mix cultures were tested for Cas9 cleavage by performing a Surveyor assay. The region of interest was amplified by PCR using the primers listed (Table 2: Primers). The Surveyor assay was performed according to the manufacturer (Integrated DNA Technologies). The mix cultures were used for experiments and to generate single-cell clones by limited dilution. Single-cell clones were analyzed by sequencing and clones with a bi-allelic shift in the open reading frame generating a premature STOP codon were chosen.

\section{Cell culture and treatment}

HCT116 $p 53^{-/-}$, HCT116 $p 53^{+/+}$(kindly provided by Bert Vogelstein) and HCT116 (ATCC) were maintained in DMEM containing 10\% FCS and Penicillin/Streptomycin (P/S). FL5.12 and BaF3 were maintained in RPMI1640 with $10 \%$ FCS, P/S and $1 \mu \mathrm{g} / 1$ recombinant IL-3. To generate IL-2-dependent lymphoid cells, lymph node cells from three $\mathrm{C} 57 / \mathrm{Bl} 6$ mice were isolated and activated by
$20 \mathrm{ng} / \mathrm{ml}$ PMA and $0.5 \mu \mathrm{g} / \mathrm{ml}$ ionomycin for $48 \mathrm{~h}$ in RPMI-1640 with $10 \%$ FSC and P/S. The cells were then cultured in the medium containing $100 \mathrm{U} / \mathrm{ml} \mathrm{recombi-}$ nant IL-2 for $24 \mathrm{~h}$ and then used for experiments. For growth factor withdrawal, cells were washed twice with $50 \mathrm{ml}$ PBS and resuspended in RPMI-1640 with 10\% FCS and $\mathrm{P} / \mathrm{S}$.

\section{Flow cytometry}

For apoptosis quantification by flow cytometry, BaF3 or FL5.12 were deprived of IL-3 in the presence or absence of CT98014 $(0.75 \mu \mathrm{M})$ for $18 \mathrm{~h}$ or as indicated. Activated lymphocytes were deprived of IL- 2 in the presence or absence of CT98014 $(0.75 \mu \mathrm{M})$ for $22 \mathrm{~h}$. The cells were washed once with Annexin-V Binding Buffer (10 mM HEPES, $150 \mathrm{mM} \mathrm{NaCl}, 150 \mu \mathrm{M} \mathrm{MgCl}, 2.5 \mathrm{mM} \mathrm{CaCl}_{2}$ ) stained for $15 \mathrm{~min}$ in the dark with Annexin-V-FITC or Annexin-V-APC in the same buffer. The fraction of Annexin-V-positive cells was measured using a FACS Calibur (BD Bioscience) or FACS LSRII (BD Bioscience).

\section{Western blotting}

Cells were harvested and washed with ice-cold PBS. Cells pellets were lysed for $5 \mathrm{~min}$ on ice with lysis buffer (20 mM Tris- $\mathrm{HCl} \mathrm{pH} 7.5,150 \mathrm{mM} \mathrm{NaCl}, 1 \%$ Triton X$100,1 \times$ protease inhibitor cocktail (Roche), phosphatase inhibitor cocktail 1 (1:50, Sigma-Aldrich), MG132 (20 $\mu \mathrm{M}$, Alexis Biochemicals)) or by nuclear fractionation as described $^{6}$. Lysates were cleared by centrifugation at $16.100 \times g$ for $10 \mathrm{~min}, 4^{\circ} \mathrm{C}$. Protein concentration was determined using Bradford reagent (Bio-Rad). Lämmli buffer was added to $10-100 \mu \mathrm{g}$ of protein lysate and samples were boiled at $95^{\circ} \mathrm{C}$ for $5 \mathrm{~min}$. In some cases, the same amount of lysate was loaded on a second gel to probe for several antibodies. Proteins were separated by SDS-PAGE and transferred to nitrocellulose membranes. The membranes were blocked in 3\% dry milk in TBSTween20 (0.1\%) (TBS-T) and then incubated at $4{ }^{\circ} \mathrm{C}$ overnight with the primary antibody diluted in $3 \%$ dry milk in TBS-T. The membranes were washed three times with TBS-T, incubated for $1 \mathrm{~h}$ with horseradish peroxidase (HRP)-conjugated secondary antibody at room temperature and washed three times with TSB-T. Band visualization was achieved using Super Signal West Pico Chemiluminescent Substrate (Thermo Scientific, Waltham, USA) and the Fusion Solo imaging system (Vilber Lourmat, Eberhardzell, Germany).

\section{Luciferase reporter assay}

293T HEK cells in 24-well plates were transfected with a total amount of $1 \mu \mathrm{g}$ DNA by Attractene. A renilla luciferase expression plasmid (Promega, Madison, WI, USA) as internal control, a luciferase reporter plasmid driven by a $1.7 \mathrm{~kb}$ Puma promotor region $(-1200$ to +500 from 
ATG) containing a conserved binding site for FOXO3A (CAAACAAT or mutated to CAGGGAAT) ${ }^{25}$ and FOXO3A-TM (T32A, S253A, S315A) pcDNA3.1 expression plasmid were used in a 1:1:1 ratio. Empty vector was added as needed to keep the total DNA constant. Eight hours after transfection DMSO or CT98014 $(0.75 \mu \mathrm{M})$ were added. Cells were lysed using $100 \mu \mathrm{l}$ luciferase-lysis buffer (50 mM Tris-phosphate $\mathrm{pH} 7.8,250 \mathrm{mM} \mathrm{KCl}, 10 \%$ Glycerol, $0.1 \%$ NP-40), lysates cleared by centrifugation at $16.100 \times g$ for $5 \mathrm{~min}, 4^{\circ} \mathrm{C}$. A volume of $10 \mu \mathrm{l}$ of the lysate was added in two black 96-well plates, renilla buffer $(25$ $\mathrm{mM}$ Tris-phosphate, $100 \mathrm{mM} \mathrm{NaCl}, 1 \mathrm{mM}$ EDTA, 0.05 $\mathrm{mM}$ Coelenterazin) or luciferase buffer $(25 \mathrm{mM}$ Trisphosphate, $10 \mathrm{mM} \mathrm{MgSO}_{4}, 2 \mathrm{mM}$ ATP, $0.05 \mathrm{mM}$ Luciferin) were auto-injected and firefly and renilla luciferase activities were determined by a plate reader.

\section{Immunoprecipitation}

293T HEK cells of a $78 \mathrm{~cm}^{2}$ culture plate were transfected by PEI with pECE-FLAG-Foxo3a or pECE-FLAGFOXO3A-TM (gifts from Michael Greenberg (Addgene plasmid \# 8360 and \# 8361) ${ }^{26}$ ) and treated with CT98014 $(0.75 \mu \mathrm{M}) 8 \mathrm{~h}$ later. The next day, the cells were lysed in 1 $\mathrm{ml}$ lysis buffer (20 mM Tris- $\mathrm{HCl} \mathrm{pH} 7.5,150 \mathrm{mM} \mathrm{NaCl}$, $1 \%$ Triton X-100, $1 \times$ protease inhibitor cocktail (Roche), phosphatase inhibitor cocktail 1 (1:50, Sigma-Aldrich), MG132 $(20 \mu \mathrm{M}$, Alexis Biochemicals)). A volume of $20 \mu \mathrm{l}$ were kept as input control and the remaining lysate was rotated for $2 \mathrm{~h}$ with $20 \mu \mathrm{l}$ FLAG-M2 agarose affinity beads (Sigma Aldrich). Beads were washed three times with lysis buffer for $3 \mathrm{~min}$. Immunoprecipitates were eluted in $50 \mu \mathrm{l}$ lysis buffer with $3 \times$ FLAG $^{\circledast}$ peptide $(150 \mathrm{ng} / \mu \mathrm{l}$, SigmaAldrich) by rotation for $30 \mathrm{~min}$ at $4{ }^{\circ} \mathrm{C}$. Eluates were then subjected to western blotting.

\section{RT-PCR}

Total RNA was extracted using Trizol (Invitrogen, Carlsbad, CA, USA) according to the manufacturer's protocol. $2 \mu \mathrm{g}$ RNA were transcribed into cDNA using the SuperScript First Strand Synthesis Kit (Invitrogen). Transcript levels of Puma and L32 were quantified by quantitative RT-PCR on an CFX96TM Real Time System thermocycler (Bio-Rad) with SYBR Green (Eurogentec, Lüttich, Belgium). The following primers were used: mPuma: 5'-GCCCAGCAGCACTTAGAGTC-3' and 5'GGTGTCGATGCTGCTCTTCT-3'; mL32: 5'-TTAAGCGAAACTGGCGGAAAC- $3^{\prime}$ and $5^{\prime}$-TTGTTGGTCCCATAACCGATG-3'.

\section{Re-expression of FOXO3A}

Production of retroviral particles and infection were performed as described above for lentiviral particles, but using $1.5 \mu \mathrm{g}$ Hit60, $1.5 \mu \mathrm{g}$ pVSV-G (Clontech) and either
$1.5 \mu \mathrm{g}$ pLXIN-hFOXO3A, plXIN-hFOXO3A-TM, or pLXIN-emtpy. Only BaF3 expressing lentiCRISPRv2 gRNA \#1 targeting mouse Foxo3a were used, as human Foxo $3 a$ is also targeted by mouse Foxo3a gRNA \#2. Selection was achieved with $50 \mathrm{ng} / \mathrm{mL}$ Geneticin $^{\mathrm{nm}}$ (ThermoFisher, Waltham, MA, USA). Experiments were performed immediately after successful selection.

\section{Overexpression of FOXO3A in HCT116 $p 53^{-1-}$}

HCT116 $p 53^{-/-}$in six-well plates were transfected by Lipofectamine $^{\circledast} 2000$ with pECE-FLAG-FOXO3A or empty vector according to the manufacturer's protocol. Twenty-four hours after transfection, the cells were treated with GDC-0941 $(10 \mu \mathrm{M})$ for $5 \mathrm{~h}$ and then subjected to western blotting.

\section{Statistical analysis}

All data are shown as mean \pm S.D. in case of technical replicates and as mean $\pm 95 \%$ confidence interval in case of biological replicates. The GraphPad Prism 5 software (GraphPad Software Inc., La Jolla, CA, USA) was applied for statistical analysis using the one-way ANOVA with post hoc Tukey's multiple comparison test. $P \leq 0.05$ was regarded as the threshold value for statistical significance. $* P \leq 0.05 ; * * P \leq 0.01 ; * * * * 0.001$.

\section{Acknowledgements \\ We thank Martin Schuler for the p53-ER ${ }^{\text {TAM }}$ plasmid, Andreas Hecht for advice and discussions, Karin Neubert for excellent technical assistance and Celia Jakob for initial experiments. This study was supported by Grants Ma 1967/1 and Ma 1967/2 from the Deutsche Forschungsgemeinschaft to U.M., Grants 109199, 107397, and 112140 from the Deutsche Krebshilfe to U.M., by funding from the Centre for Biological Signalling Studies (BIOSS, EXC-294), Freiburg, Germany to C.B. and U.M., and the Spemann Graduate School of Biology and Medicine (SGBM, GSC-4), Freiburg, Germany, to C.B. and U.M. both funded by the Excellence Initiative of the German Federal and State Governments, Germany.}

\begin{abstract}
Author details
${ }^{1}$ Institute of Molecular Medicine and Cell Research, Faculty of Medicine, AlbertLudwigs-University of Freiburg, Freiburg, Germany. ${ }^{2}$ Spemann Graduate School of Biology and Medicine (SGBM), Albert-Ludwigs-University of Freiburg, Freiburg, Germany. ${ }^{3}$ Faculty of Biology University of Freiburg Schänzlestrasse 1, 79104 Freiburg, Germany. ${ }^{4}$ BIOSS, Centre for Biological Signaling Studies, Hebelstrasse 2, 79104 Freiburg, Germany. ${ }^{5}$ Functional Genomics and Cancer, Institut de Génétique et de Biologie Moléculaire et Cellulaire (IGBMC), INSERM U964, CNRS UMR 7104, Université de Strasbourg, 1 rue Laurent Fries, Illkirch 67404, France
\end{abstract}

Conflict of interest

The authors declare that they have no conflict of interest.

Publisher's note

Springer Nature remains neutral with regard to jurisdictional claims in published maps and institutional affiliations.

Supplementary Information accompanies this paper at https://doi.org/ 10.1038/s41419-018-0502-4. 
Received: 18 December 2017 Revised: 9 March 2018 Accepted: 15 March 2018

Published online: 23 April 2018

\section{References}

1. Llambi, F. et al. A unified model of mammalian BCL-2 protein family interactions at the mitochondria. Mol. Cell 44, 517-531 (2011).

2. Brunet, A. et al. Akt promotes cell survival by phosphorylating and inhibiting a Forkhead transcription factor. Cell 96, 857-868 (1999).

3. Maurer, U., Preiss, F., Brauns-Schubert, P., Schlicher, L. \& Charvet, C. GSK-3 - at the crossroads of cell death and survival. J. Cell Sci. 127, 1369-1378 (2014).

4. Pap, M. \& Cooper, G. M. Role of glycogen synthase kinase-3 in the phosphatidylinositol 3-Kinase/Akt cell survival pathway. J. Biol. Chem. 273, 19929-19932 (1998)

5. You, H. et al. FOXO3a-dependent regulation of Puma in response to cytokine/ growth factor withdrawal. J. Exp. Med. 203, 1657-1663 (2006).

6. Charvet, C. et al. Phosphorylation of Tip60 by GSK-3 determines the induction of PUMA and apoptosis by p53. Mol. Cell 42, 584-596 (2011).

7. Ambacher, K. K. et al. The JNK- and AKT/GSK3ß- signaling pathways converge to regulate Puma induction and neuronal apoptosis induced by Trophic Factor deprivation. PLOS ONE 7, e46885 (2012).

8. Huo, X. et al. GSK3 protein positively regulates type I insulin-like growth factor receptor through Forkhead transcription factors FOXO1/3/4. J. Biol. Chem. 289, 24759-24770 (2014).

9. Maurer, U., Charvet, C., Wagman, A. S., Dejardin, E. \& Green, D. R. Glycogen synthase kinase-3 regulates mitochondrial outer membrane permeabilization and apoptosis by destabilization of MCL-1. Mol. Cell 21, 749-760 (2006).

10. Nakano, K. \& Vousden, K. H. PUMA, a novel proapoptotic gene, is induced by p53. Mol. Cell 7, 683-694 (2001).

11. Yu, J., Zhang, L., Hwang, P. M., Kinzler, K. W. \& Vogelstein, B. PUMA induces the rapid apoptosis of colorectal cancer cells. Mol. Cell 7, 673-682 (2001).

12. Sykes, S. M. et al. Acetylation of the p53 DNA-binding domain regulates apoptosis induction. Mol. Cell 24, 841-851 (2006).
13. Tang, Y., Luo, J., Zhang, W. \& Gu, W. Tip60-dependent acetylation of p53 modulates the decision between cell-cycle arrest and apoptosis. Mol. Cell 24 827-839 (2006).

14. Tang, Y., Zhao, W., Chen, Y., Zhao, Y. \& Gu, W. Acetylation is indispensable for p53 activation. Cell 133, 612-626 (2008).

15. Lindner, S. E. et al. Increased leukocyte survival and accelerated onset of lymphoma in the absence of MCL-1 S159-phosphorylation. Oncogene 33, 5221-5224 (2014)

16. Stahl, M. et al. The Forkheadtranscription factor FoxO regulates transcription of p27Kip1 and Bim in response to IL-2. J. Immunol. 168 5024-5031 (2002).

17. Ekert, P. G. et al. Cell death provoked by loss of interleukin-3 signaling is independent of Bad, Bim, and PI3 kinase, but depends in part on Puma. Blood 108, 1461-1468 (2006).

18. Ekoff, $\mathrm{M}$. et al. The $\mathrm{BH} 3$-only protein Puma plays an essential role in cytokine deprivation-induced apoptosis of mast cells. Blood 110, 3209-3217 (2007).

19. Nayak, G. \& Cooper, G. M. p53 is a major component of the transcriptional and apoptotic program regulated by PI 3-kinase/Akt/GSK3 signaling. Cell Death Dis. 3, e400 (2012).

20. Jabbour, A. M. et al. Myeloid progenitor cells lacking p53 exhibit delayed upregulation of Puma and prolonged survival after cytokine deprivation. Blood 115, 344-352 (2010).

21. Jabbour, A. M. et al. P53-dependent transcriptional responses to Interleukin-3 signaling. PLoS ONE 7, e31428 (2012).

22. Cahill, C. M. et al. Phosphatidylinositol 3-Kinase signaling inhibits DAF-16 DNA binding and function via 14-3-3-dependent and 14-3-3-independent pathways. J. Biol. Chem. 276, 13402-13410 (2001).

23. Terragni, J. et al. The E-box binding factors Max/Mnt, MITF, and USF1 act coordinately with FoxO to regulate expression of proapoptotic and cell cycle control genes by phosphatidylinositol 3-kinase/akt/glycogen synthase kinase 3 signaling. J. Biol. Chem. 286, 36215-36227 (2011).

24. Sanjana, N. E., Shalem, O. \& Zhang, F. Improved vectors and genome-wide libraries for CRISPR screening. Nat. Methods 11, 783-784 (2014).

25. You, H., Yamamoto, K. \& Mak, T. W. Regulation of transactivation-independent proapoptotic activity of p53 by FOXO3a. Proc. Natl Acad. Sci. USA 103 9051-9056 (2006)

26. Brunet, A. et al. Stress-dependent regulation of FOXO transcription factors by the SIRT1 deacetylase. Science 303, 2011-2015 (2004). 